\title{
Metadiffusers for quasi-perfect and broadband sound diffusion
}

Cite as: Appl. Phys. Lett. 119, 044101 (2021); https://doi.org/10.1063/5.0053413

Submitted: 07 April 2021 . Accepted: 14 July 2021. Published Online: 26 July 2021

E. Ballestero, (iD N. Jiménez, (iD J.-P. Groby, (iD H. Aygun, (D) S. Dance, and (iD V. Romero-García

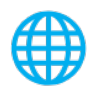

\section{ARTICLES YOU MAY BE INTERESTED IN}

Remote triggering of air-gap discharge by a femtosecond laser filament and postfilament at distances up to $80 \mathrm{~m}$

Applied Physics Letters 119, 041103 (2021); https://doi.org/10.1063/5.0057544

Constructing light with high precision using source coherence

Applied Physics Letters 119, 041102 (2021); https://doi.org/10.1063/5.0057666

2.6 K VCSEL data link for cryogenic computing

Applied Physics Letters 119, 041101 (2021); https://doi.org/10.1063/5.0054128

耳QBLOX

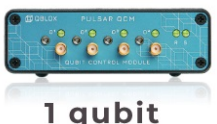

AlP
Shorten Setup Time

Auto-Calibration

More Qubits

Fully-integrated

Quantum Control Stacks

Ultrastable DC to $18.5 \mathrm{GHz}$

Synchronized $<<1$ ns

Ultralow noise

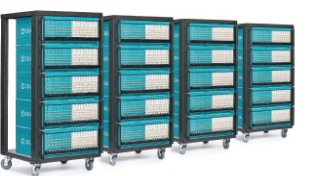

100s qubits

visit our website > 


\title{
Metadiffusers for quasi-perfect and broadband sound diffusion
}

\author{
Cite as: Appl. Phys. Lett. 119, 044101 (2021); doi: 10.1063/5.0053413 \\ Submitted: 7 April 2021 . Accepted: 14 July 2021 . \\ Published Online: 26 July 2021
}

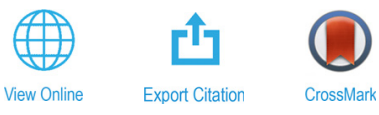

E. Ballestero, ${ }^{1, a)}$ (D) N. Jiménez, ${ }^{2}$ (D) J.-P. Groby, ${ }^{3}$ (D) H. Aygun, ${ }^{7}$ (D) S. Dance, ${ }^{1}$ (D) and V. Romero-García ${ }^{3}$ (D)

\begin{abstract}
AFFILIATIONS
'The Acoustics Group, London South Bank University, 103 Borough Road, London SE1 OAA, United Kingdom

${ }^{2}$ Instituto de Instrumentación para Imagen Molecular, Consejo Superior de Investigaciones Científicas (CSIC), Universitat Politècnica de València, Camino de Vera s/n, 46022 València, Spain

${ }^{3}$ Laboratoire d'Acoustique de I'Université du Mans (LAUM), UMR CNRS, 6613, Institut d'Acoustique-Graduate School (IA-CS), CNRS, Le Mans Unversité, Avenue Olivier Messiaen, 72085 Le Mans Cedex 9, France
\end{abstract}

${ }^{\text {a) }}$ Author to whom correspondence should be addressed: ballesteroeric@outlook.com

\begin{abstract}
Sound diffusion refers to the ability of a surface to evenly scatter sound energy in both time and space. However, omni-directional radiation of sound, or perfect diffusion, can be impractical or difficult to reach under traditional means. This is due to the considerable size required, and the lack of tunability, of typical quarter-wavelength scattering strategies necessary for producing the required complexity of the surface acoustic impedance. As such, it can be a challenge to design sound diffusing structures that can display near perfect diffusion performance within slim dimensions. In this work, we propose a method for obtaining quasi-perfect and broadband sound diffusion coefficients using deep-subwavelength acoustic diffusers, i.e., metadiffusers. The relation among the geometry of the metasurface, the bandwidth, and the diffusion performance is analytically and numerically studied. For moderate bandwidths, around $1 / 3$ of an octave, the method results in nearly perfect sound diffusion, while for a bandwidth of 2.5 octaves, a normalized diffusion coefficient of 0.8 was obtained using panels $1 / 30$ th thinner than traditional phase-grating designs. The ratio between the wavelength and the size of the unit cell was identified as a limitation of the performance. This work demonstrates the versatility and effectiveness of metadiffusers to generate diffuse reflections outperforming those of classical sound diffusers.
\end{abstract}

Published under an exclusive license by AIP Publishing. https://doi.org/10.1063/5.0053413

The theory behind sound diffusion is based on the physical principles of wave diffraction. The phenomenon can commonly be defined through the Helmholtz-Kirchhoff integral formulation, which allows to describe the output scattered wave field generated by a diffracting object given a certain input wave field. ${ }^{1}$ In acoustics, sound diffusers are often sought for scattering the reflected sound field; it may be for dispersing it or focusing it toward specific directions. In this work, an emphasis is given to diffusers that radiate waves omnidirectionnally, resulting in a perfect diffusion of the reflected sound field.

Sound diffusion can be reached through a specific spatial distribution of the impedance along the surface, leading to a profile of the reflection coefficient. These locally reacting surfaces are often termed Schröeder diffusers. ${ }^{2}$ Usually, Schröeder diffusers make use of quarterwavelength resonators (QWRs), in order to achieve a phase profile of the reflection coefficient following a numerical sequence with a flat Fourier transform. However, this strategy often results in bulky structures for large wavelengths, as the maximum phase shift of the reflection coefficient occurs at $L \approx c_{0} / 4 f$, where $f$ is the working frequency, $L$ is the depth of the QWR, and $c_{0}$ is the speed of sound in air. This size limitation along with the fact that QWRs do not offer a great tunability of the magnitude and phase of the reflection coefficient limit the use of Schröeder diffusers, where adjustable phase and magnitude behaviors are required.

Through the last decades, several diffuser strategies have been proposed in order to enhance the major inconvenience of Schröeder diffusers. Folding strategies were proposed to minimize space between slits. ${ }^{3,4}$ Hunecke ${ }^{5}$ proposed a closed QWR model by adding perforated or micro-perforated sheets ${ }^{4,6}$ for lowering the resonant frequencies along with the low cut off design frequency. More recently, sonic crystals (SCs) were used to construct volumetric acoustic diffusers. ${ }^{7,8}$ In addition, optimized sound diffusers made of slotted panels incorporating two dimensional Helmholtz resonators ${ }^{9}$ (HRs) instead of QWRs have already been commercialized. By using HRs, the resonance frequency of each slit can be down-shifted, thus extending the diffusion 
bandwidth. This idea has recently been revisited by using metamaterial-inspired strategies, allowing the design of metasurfaces presenting simultaneously efficient diffusion properties and deep-subwavelength dimensions. In 2017, Zhu et al. ${ }^{10}$ revisited the problem to design an ultrathin quadratic residue diffuser (QRD) using a planar array of HRs focused on the low frequency range. Also in 2017, the concept of metadiffusers was proposed by Jiménez et al. ${ }^{11}$

Metadiffusers are rigidly backed slotted panels based on slowsound metamaterials, ${ }^{12}$ where each slit is loaded by an array of HRs. That way, strong dispersion is introduced and the effective sound speed inside each slit is drastically reduced in the low frequency regime. ${ }^{12,13}$ This causes the quarter-wavelength resonance to be shifted into the deep-subwavelength regime, therefore strongly reducing the effective thickness of the panel. ${ }^{14-16}$ The geometry variables of each metadiffuser design can be calculated following an optimization procedure for replicating the reflection coefficient profile corresponding to a given numerical sequence at a particular frequency. ${ }^{11}$ As such, many designs were proposed based on numerical sequences that already exist for traditional diffusers. Recently, the diffusion characteristics of a 3Dprinted quadratic residue metadiffuser (QRM) were experimentally measured and compared to analytical and numerical results, ${ }^{17}$ where good agreement was found.

However, although all the above-mentioned metadiffuser designs present high values of sound diffusion coefficient, their potential to present omnidirectional sound scattering, referred here as perfect diffusion, is not discussed. Moreover, contrary to typical optimizations of acoustic metasurfaces for focusing ${ }^{18}$ or redirecting ${ }^{19}$ the waves in a selected direction, here we discuss the design of a metasurface with perfect omnidirectional wave diffusion; the latter being particularly relevant for acoustic purposes. In this work, we propose a method for maximizing quasi-perfect diffusion (QPD) of sound in narrow frequency bands based on acoustic metadiffusers. Additionally, we also provide an optimization study underlining the conditions required for the system to reach high broadband sound diffusion over a significant portion of the audible frequency range. Both types of structures work within deep-subwavelength dimensions much thinner than their typical QWR equivalents.

Following Fraunhofer's diffraction theory as an approximation of the Helmholtz-Kirchhoff integral equation, the scattered sound pressure distribution over a reflecting surface can usually be described by a spatial Fourier transform of the complex reflection coefficient. In this case, the far-field scattered pressure distribution at normal incidence, $p_{s}(\theta, \phi)$, of a reflecting rectangular surface with a spatially dependent reflection coefficient, $R(x, y)$, of size $2 a$ and $2 b$ in the $x$ and $y$ directions, respectively, can be calculated using the Fraunhofer integral ${ }^{20}$

$$
p_{s}(\theta, \phi)=\int_{-a}^{a} \int_{-b}^{b} R(x, y) e^{i k(x \sin \theta \sin \phi+y \sin \theta \cos \phi)} \mathrm{d} x \mathrm{~d} y,
$$

where $\theta$ and $\phi$ are the elevational and azimuthal angles, respectively.

The diffusion coefficient of a surface rates the uniformity of the aforementioned scattered sound field. The directional diffusion coefficient, ${ }^{21} \delta_{\psi}$, produced when a sound diffuser is illuminated by a plane wave at the incident angle $\psi=\left(\theta^{\prime}, \phi^{\prime}\right)$ (primed superscripts denoting incident angles) can be estimated from the hemispherical distribution

$$
\delta_{\psi}=\frac{\left[\iint I_{s}(\theta, \phi) \mathrm{d} S\right]^{2}-\iint I_{s}^{2}(\theta, \phi) \mathrm{d} S}{\iint I_{s}^{2}(\theta, \phi) \mathrm{d} S},
$$

where $I_{s}(\theta, \phi) \propto\left|p_{s}(\theta, \phi)\right|^{2}$ is proportional to the scattered intensity. The integration is performed over a hemispherical surface $(-\pi / 2$ $\leq \theta \leq \pi / 2$ and $0<\phi<2 \pi)$, where $\mathrm{d} S=\mathrm{d} \theta \mathrm{d} \phi$. This coefficient must be normalized to that of a plane reflector, $\delta_{\text {flat }}$, so as to eliminate the diffracting effect caused by the finite size of the structure, i.e., $\delta_{n, \psi}=\left(\delta_{\psi}-\delta_{\text {flat }}\right) /\left(1-\delta_{\text {flat }}\right)$. We analyze here the $2 \mathrm{D}$ case of normal plane wave incidence in the elevational plane only, i.e., $\theta^{\prime}=0$ and $\delta_{n, \psi} \equiv \delta_{0}$.

Analytical surface reflection coefficients required to calculate the far-field sound scattering and frequency dependent diffusion coefficients were obtained with the transfer matrix method ${ }^{11}$ (TMM), which relates the acoustic pressures and normal particle velocities at the extremities of a one-dimensional acoustic system. Numerical simulations were computed using the finite element method (FEM) in COMSOL Multiphysics ${ }^{\circledR}$. The surface was installed at the center of a circular domain filled with air, surrounded by a concentric perfectly matched layer (PML) with a far-field boundary condition at the boundary of the air domain to simulate the radiation condition.

Whereas the geometry of the previous metadiffusers was being optimized to produce a reflection coefficient profile fitting a particular numerical sequence at a certain frequency, a different approach to design metadiffusers for QPD is proposed here: we look for the geometry of the metadiffuser that directly maximizes the normalized diffusion coefficient at the target frequency. The optimization paradigm relies on the same constrained minimization algorithm used in Ref. 11, where the cost function $\varepsilon_{Q P D}=1-\delta_{n, \psi}(f)$ is minimized so that the normalized diffusion coefficient $\left(0 \leq \delta_{n, \psi}(f) \leq 1\right)$ would tend to unity at the target frequency.

The total surface of the metadiffuser, of length $D$, is divided in $N$ unit cells of periodicity $a_{x}=D / N$. The $j$ th unit cell presents a slit of thickness $L^{j}$ and width $h^{j}$ loaded with $M$ resonators, which are characterized by the lengths of the neck and cavity, $l_{n}^{j}$ and $l_{c}^{j}$, respectively, and the widths of the neck and the cavity, $w_{n}^{\prime}$ and $w_{c}^{j}$, respectively. In such a way, the reflection coefficient presents a profile along $x$ with $N$ different values over the total length of the surface. This profile is then optimized with the previously described methodology.

Figure 1(a) shows the geometry obtained from the optimization of an $N=11, M=1, D=1.32 \mathrm{~m}$, and $L=3 \mathrm{~cm}$ QPD-metadiffuser, as well as the target phase of the reflection coefficient required for QPD at $500 \mathrm{~Hz}$. The spatial Fourier transform of such a phase profile provides a constant scattered amplitude in space. A large panel width $D=1.32 \mathrm{~m}$ has been taken here in order to avoid the low frequency diffraction regime that would otherwise occur at dimensions close to $\lambda \approx 0.7 \mathrm{~m}$ at $500 \mathrm{~Hz}$. The geometry dimensions of the QPD can be found in Table I. Figure 1(b) compares the analytical and numerical normalized diffusion coefficients considering visco-thermal losses ${ }^{22}$ of two QPD-metadiffusers of the same width $D=1.32 \mathrm{~m}$, one with $N=11$ slits aimed for perfect diffusion at $500 \mathrm{~Hz}$ and another with $N=20$ slits tuned at $1.5 \mathrm{kHz}$. In addition, a similarly optimized $N=11$ slits quarter-wavelength diffuser (QWD) made for replicating the complex surface impedance required for QPD is displayed alongside the two previous metadiffusers. This enables the comparison of 

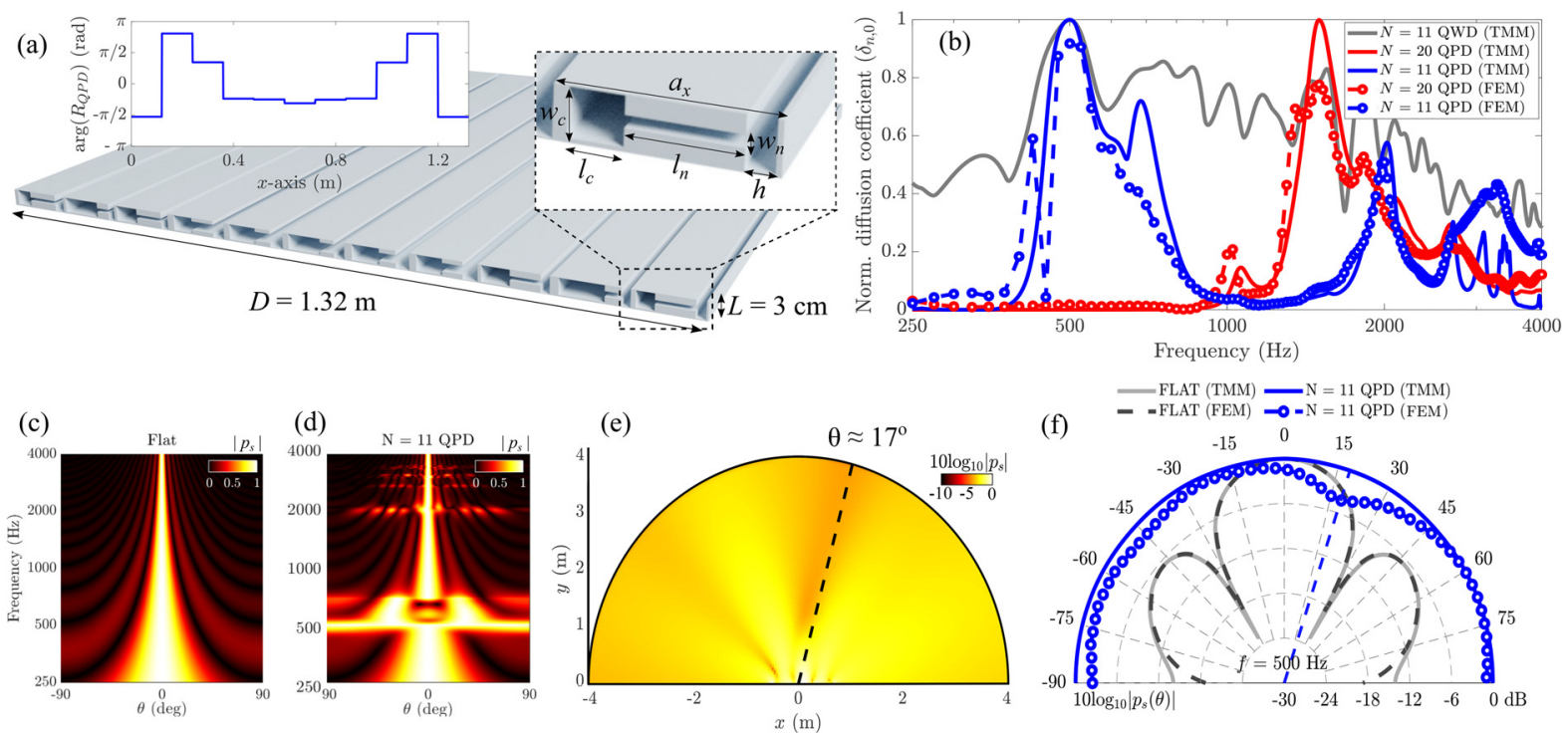

FIG. 1. (a) Geometry of the $N=11$ slits quasi-perfect diffusion (QPD) metadiffuser design with $L=3 \mathrm{~cm}, M=1$, and $D=1.32 \mathrm{~m}$. (inset) Target phase of the reflection coefficient at $500 \mathrm{~Hz}$. (b) Normalized diffusion coefficients of an $N=11$ slit QPD (blue), an $N=20$ slit QPD (red), and an optimized $N=11$ quarter wavelength diffuser (QWD) (grey). (c) and (d) Far-field scattered sound energy against frequency for a flat surface and $N=11$ QPD. (e) Near-field of the $N=11$ QPD obtained through FEM. (f) Far field scattered sound energy at $f=500 \mathrm{~Hz}$ for the $N=11 \mathrm{QPD}$ and flat reference surface of the same width.

diffusion properties between the equally optimized QPD structures. Figure 1(b) shows that the overall shapes of the analytical and numerical curves are in fair agreement with the frequency axis despite some discrete discrepancies. These can be explained by the limitation of analytical assumptions where the evanescent coupling between slits, affecting the local distribution of the acoustic impedance at the surface, is not accounted for. Other assumptions, such as higher order lateral modes for the HRs, are not accounted for as well and might explain some high frequency analytical-to-numerical disparities. Nonetheless, the numerical curve for the $N=11$ QPD displays an excellent normalized diffusion peak value of $\delta_{n, 0}=0.92$ at $500 \mathrm{~Hz}$, which is fairly close

TABLE I. Geometrical parameters for the jth well of the $N=11$ QPD-metadiffuser. $h$ is the width of the slit, and $I_{n}, I_{c}, w_{n}$, and $W_{c}$ are the lengths and widths of the neck and the cavity of the HR, respectively.

\begin{tabular}{lcccccc}
\hline \hline$j$ & $L(\mathrm{~mm})$ & $h(\mathrm{~mm})$ & $l_{n}(\mathrm{~mm})$ & $l_{c}(\mathrm{~mm})$ & $w_{n}(\mathrm{~mm})$ & $w_{c}(\mathrm{~mm})$ \\
\hline 1 & 30 & 15.5 & 60.7 & 29.1 & 7.2 & 28 \\
2 & 30 & 14.2 & 25.7 & 73.2 & 9.2 & 28 \\
3 & 30 & 14.9 & 50.9 & 48.5 & 7.5 & 28 \\
4 & 30 & 26.0 & 35.4 & 46.3 & 14.4 & 28 \\
5 & 30 & 24.8 & 30.1 & 51.4 & 13.9 & 28 \\
6 & 30 & 20.0 & 26.4 & 62.0 & 16.1 & 28 \\
7 & 30 & 25.1 & 30.2 & 52.8 & 15.3 & 28 \\
8 & 30 & 22.7 & 44.7 & 39.2 & 14.3 & 28 \\
9 & 30 & 14.8 & 53.9 & 45.6 & 7.4 & 28 \\
10 & 30 & 13.7 & 25.6 & 74.5 & 9.6 & 28 \\
11 & 30 & 19.6 & 59.4 & 30.7 & 7.2 & 28 \\
\hline \hline
\end{tabular}

to the analytical design value of $\delta_{n, 0}=0.99$. A very good agreement is shown at the second diffusion peak near $2 \mathrm{kHz}$, probably due to the second resonance mode of the slits. Higher diffusion peaks around $3 \mathrm{kHz}$ seem to have more discrepancies between the different methods but reside within the same frequency range.

The $N=11$ QWD shown in Fig. 1(b) displays a normalized diffusion coefficient as good as the $N=11$ QPD-metadiffuser at $500 \mathrm{~Hz}$, i.e., $\delta_{n, 0}=0.99$. However, this performance comes at a steep price in terms of slit depth, resulting in a maximum depth of $81 \mathrm{~cm}$, which is 27 times larger than the $N=11$ QPD-metadiffuser of $3 \mathrm{~cm}$.

The $N=20$ metadiffuser tuned for $1.5 \mathrm{kHz}$ shown in Fig. 1(b) has been made with a higher number of slits in order to increase the modularity of the panel and better adapt to the more complex surface impedance that is required to reach QPD in a higher frequency regime for the same panel width. In such a case, a numerical normalized diffusion peak value of $\delta_{n, 0}=0.77$ is obtained at $1.5 \mathrm{kHz}$. Although the analytical reflection coefficient at such frequencies produces QPD $\left(\delta_{n, 0}=0.99\right)$, it appears that the numerical results do not match so well. This is probably due to the effects not considered in the theory that are likely to arise in high frequency regimes, i.e., higher order modes and evanescent coupling between slits. The numerical amplitude peak at $1.5 \mathrm{kHz}$ could be mitigated by directly optimizing the numerical model for generating omnidirectional scattering. Physically, reaching near-perfect diffusion values at high frequencies $(D / \lambda \gg 1)$ can be achieved by increasing the number of slits within the same panel width. This is because the sum of the scattered waves generated by wide slits, radiating as directive pistons, cannot interfere at other directions than in normal and, thus, fail to efficiently produce uniform scattering. In addition, if the separation between slits is larger than half wavelength, secondary diffraction grating lobes emerge in the far field at angles $\beta_{q}=\sin ^{-1}(q \lambda N / D)$ with $q=1,2, \ldots$ being the diffraction 
order. To reach efficient sound diffusion, each slit would, thus, need to approximate a punctual scatterer, and the separation between them should grant the absence of diffraction grating lobes. This suggests a requirement for QPD following the relation $k a_{x}<\pi$, where $k$ is the wavenumber.

Figure 1(c) shows the far-field scattered sound energy against frequency for a flat reflector, where natural diffraction lobes generated by wave interference are illustrated by the increasing number of high and low polar energy distributions along frequency. Alternatively, the QPD of the $N=11$ metadiffuser is illustrated in Fig. 1(d) by a thin and uniform energy band around $500 \mathrm{~Hz}$. Figure 1 (e) shows the intricacies of the QPD in the near field obtained numerically, in which a darker field amplitude area can be seen at $\theta \approx 17^{\circ}$. This is also illustrated in Fig. 1(f) that displays the scattered far-field for the same QPDmetadiffuser, where the magnitude of the normalized scattering obtained numerically at $\theta=17^{\circ}$ is $-4 \mathrm{~dB}$. The numerical polar distribution at other angles is otherwise quite uniform, resulting in high diffusion performance.

Heretofore, two scenarios of QPD-metadiffusers have been discussed, which targeted different frequencies with a specific number of slits. However, metadiffuser optimizations can be further extended to go from single frequency QPD to high broadband sound diffusion. In such a case, broadband metadiffusers are designed by modifying the optimization cost function to account for normalized diffusion coefficients held between a low and high cut frequencies, i.e., $\varepsilon_{\text {broad }}=1-\delta_{n, 0, \text { avge }}\left(n_{f}\right)$, where $\delta_{n, 0, \text { avge }}\left(n_{f}\right)=\int_{f_{\text {tiv }}}^{f_{\text {nigh }}} \delta_{n, 0} \mathrm{~d} f / n_{f}$ is the normalized diffusion coefficient averaged over $n_{f}$ frequency samples. Thus, highly efficient broadband metadiffusers can be designed where the geometry constraints $(N, D, M, L)$ remain the same as previously while the rest of the geometry (slits and HRs) would now fit the cost function $\varepsilon_{\text {broad }}$ for several frequency ranges $\Delta_{f}=\left[f_{\text {low }}: f_{\text {high }}\right]$. The broadband dimension constraints were chosen to be the same as the QPD case in order to demonstrate the adaptability of the metadiffuser for going from QPD to highly effective broadband diffusion within the same overall structure dimensions. Considering this multitude of variables, Fig. 2 emphasizes on the general rate of dependence between the average diffusion coefficient and the number of slits per panel, which was obtained over a large population of optimizations with different broadband objectives.

Figure 2(a), thus, illustrates the cross dependency of the number of slits $N$ and the frequency ranges $\Delta_{f}$ for optimizing $\delta_{n, 0, \text { avge }}$ for a metadiffuser of fixed width $D=1.32 \mathrm{~m}$. First, a trend emerges from the positive gradient of the data presented, i.e., the higher the number of slits, the more likely the diffusion coefficient would be efficiently optimized for all the different frequency ranges with a minimum limit of $N=3$ slits. This is due to the increased number of slits with which the surface can better adapt to more complex impedance requirements. This can be observed at $N=3$, where orange-red curves (wide frequency ranges) do not reach as high values as the bluer curves (narrow frequency ranges). However, at $N=13$, orange-red curves show diffusion values close to the blue curves despite the much larger frequency ranges being covered. These results allow us to clearly demonstrate that a high number of narrow slits is indeed required to efficiently modulate an omnidirectional scattering of sound over many frequencies. Not only a punctual scatterer approximation for each slit is critical but also a minimum number of slits is required so as to avoid diffraction grating lobes, the maximum spacing between each being smaller than half the wavelength considered, i.e., $a_{x}<\lambda / 2$. Thus, in a similar way to QPD, this would suggest a requirement for obtaining quasi-perfect broadband diffusion to follow the relation $k a_{x}<\pi, \forall k \in 2 \pi \Delta_{f} / c$, where $k$ is the wavenumber. Figure 2(b) shows examples of the various normalized diffusion coefficients obtained through different $\Delta_{f}$ frequency bandwidths tested. In the case of
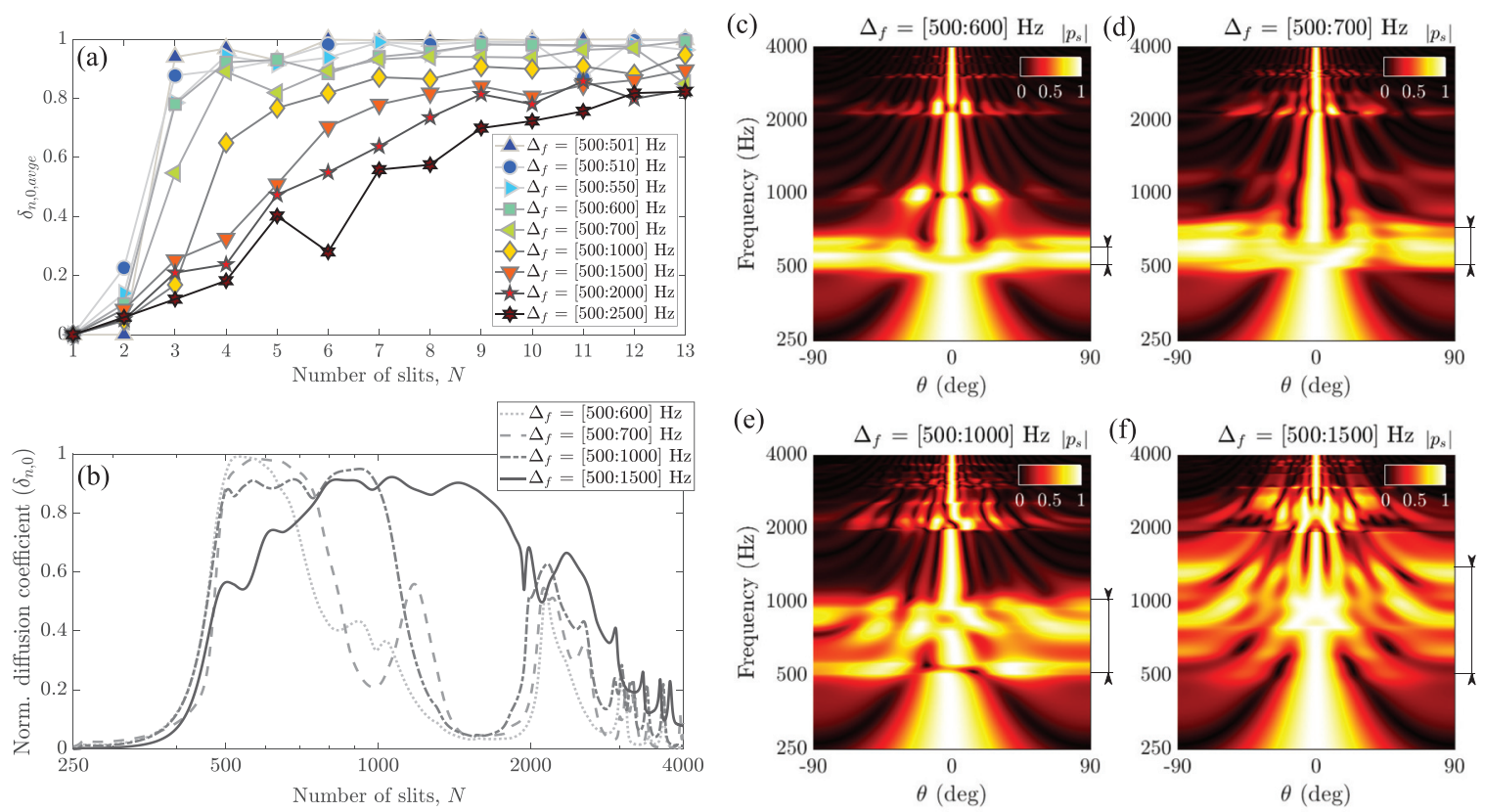

FIG. 2. (a) Cross dependency of $\delta_{n, 0 \text {,avge }}$ as a function of the number of slits $N$ and frequency ranges $\Delta_{f}$ for a metadiffuser of fixed width $D=1.32 \mathrm{~m}$. (b) Normalized diffusion coefficients of various broadband metadiffuser designs for different $\Delta_{f}$ frequency ranges. (c)-(f) Far-field scattered sound energy against frequency for various $\Delta_{f}$ frequency ranges. 
$\Delta_{f}=[500: 600] \mathrm{Hz}$, a value $\delta_{n, 0, \text { avge }}=0.98$ is achieved. This is an extremely high value considering its frequency spans over $100 \mathrm{~Hz}$. For the other cases, one can observe that as the frequency range $\Delta_{f}$ increases, $\delta_{n, 0, \text { avge }}$ decreases. Yet, the latter still remains at high values, viz., $\delta_{n, 0, \text { avge }}=0.96,0.90$, and 0.84 , for ranges $\Delta_{f}=[500: 700]$, $\Delta_{f}=[500: 1000]$, and $\Delta_{f}=[500: 1500] \mathrm{Hz}$, respectively. In addition, a diffusion peak can be outlined for all the $\Delta_{f}$ designs. In the case of $\Delta_{f}=[500: 1500] \mathrm{Hz}$, this peak provides a continuous decrease in the normalized diffusion coefficient to even higher frequencies. Figures 2(c)-2(f) show the far-field scattered sound pressure fields for the different $\Delta_{f}$ ranges selected. The fact that the diffusion coefficient gets broader along the frequency axis is displayed through a larger and more uniform angular energy band.

In this work, we have demonstrated the potential of metadiffusers for displaying quasi-perfect normalized sound diffusion coefficients within deep-subwavelength dimensions. It has been shown that the slit width and the separation between them are a major factor to account for when aiming for quasi-perfect or broadband sound diffusion due to the directive radiation of individual slits and the emergence of diffraction grating lobes. In addition, using multiple slits rather than a few enhances the flexibility to engineer the complex surface impedance, thus resulting in efficient uniform scattering. The ability to obtain such range of efficient scattered sound distributions within ultrathin dimensions, instead of larger alternatives, can be welcome when dealing with environments where space is at a premium, e.g., aerospace applications ${ }^{23}$ or orchestra pits. ${ }^{4}$ The results shown in this work demonstrate the usefulness of metadiffusers to be applied in many practical situations, where they can outshine classical solutions due to their versatility.

See the supplementary material for a brief description of the transfer matrix method (TMM) used in the modeling of metadiffusers.

The collaboration between the authors of this article was made possible thanks to the European Cooperation in Science and Technology (COST) Action CA15125-Design in Noise Reducing Materials (DENORMS). This study was financed by the Royal Opera House, Covent Garden, and the United Kingdom Acoustics Network (UKAN). The authors gratefully acknowledge the ANR-RGC METARoom (No. ANR-18-CE08-0021) project and the project HYPERMETA funded under the program Étoiles Montantes of the Région Pays de la Loire. N.J. acknowledges financial support from Generalitat Valenciana through Grant No. APOSTD/2017/042.

\section{DATA AVAILABILITY}

The data that support the findings of this study are available from the corresponding author upon reasonable request.

\section{REFERENCES}

${ }^{1}$ A. Pierce, Acoustics: An Introduction to Its Physical Principles and Applications, McGraw-Hill Series in Mechanical Engineering (McGraw-Hill Book Company, 1981).

${ }^{2}$ M. R. Schröder, J. Acoust. Soc. Am. 57, 149 (1975).

${ }^{3}$ F. P. Mechel, Acta Acust. Acust. 81, 512-527 (1995).

${ }^{4}$ T. Cox and P. D’Antonio, Build. Acoust. 10(1), 1-32 (2003).

${ }^{5}$ J. Hunecke, "Schallstreuung und schallabsorption von oberfl: Ahen aus mikroperforierten streifen," Ph.D. thesis (University of Stuttgart, 1997).

${ }^{6}$ T. Wu, T. J. Cox, and Y. Lam, J. Acoust. Soc. Am. 110, 3064 (2001).

${ }^{7}$ R. J. Hughes, J. A. Angus, T. J. Cox, O. Umnova, G. A. Gehring, M. Pogson, and D. M. Whittaker, J. Acoust. Soc. Am. 128, 2847 (2010).

${ }^{8}$ J. Redondo, R. Picó, V. J. Sánchez-Morcillo, and W. Woszczyk, J. Acoust. Soc. Am. 134, 4412 (2013).

${ }^{9}$ P. DAntonio, "Planar binary amplitude diffusor," U.S. patent 5817992 A (1998).

${ }^{10}$ Y. Zhu, X. Fan, B. Liang, J. Cheng, and Y. Jing, Phys. Rev. X 7, 021034 (2017).

${ }^{11}$ N. Jiménez, T. J. Cox, V. Romero-García, and J.-P. Groby, Sci. Rep. 7, 5389 (2017).

${ }^{12}$ J.-P. Groby, W. Huang, A. Lardeau, and Y. Aurégan, J. Appl. Phys. 117, 124903 (2015).

${ }^{13}$ A. Santillán and S. I. Bozhevolnyi, Phys. Rev. B 84, 064304 (2011).

${ }^{14}$ N. Jiménez, W. Huang, V. Romero-García, V. Pagneux, and J.-P. Groby, Appl. Phys. Lett. 109, 121902 (2016).

${ }^{15}$ N. Jiménez, V. Romero-García, V. Pagneux, and J.-P. Groby, Phys. Rev. B 95, 014205 (2017).

${ }^{16}$ N. Jiménez, V. Romero-García, V. Pagneux, and J.-P. Groby, Sci. Rep. 7, 13595 (2017).

${ }^{17}$ E. Ballestero, N. Jiménez, J.-P. Groby, S. Dance, H. Aygun, and V. RomeroGarcía, Appl. Phys. Lett. 115, 081901 (2019).

${ }^{18}$ X. Jiang, Y. Li, D. Ta, and W. Wang, Phys. Rev. B 102, 064308 (2020).

${ }^{19}$ J. Zhao, B. Li, Z. N. Chen, and C.-W. Qiu, Appl. Phys. Lett. 103, 151604 (2013).

${ }^{20}$ T. D. Rossing, F. Dunn, W. M. Hartmann, D. M. Campbell, and N. H. Fletcher, Springer Handbook of Acoustics, 1st ed. (Springer Publishing Company, Inc., 2007).

${ }^{21}$ International Organization for Standardization, ISO 17497-2:2012 AcousticsSound-Scattering Properties of Surfaces-Part 2: Measurement of the Directional Diffusion Coefficient in a Free Field (International Organization for Standardization, 2012).

${ }^{22}$ M. R. Stinson, J. Acoust. Soc. Am. 89, 550 (1991).

${ }^{23}$ L. Garcia-Raffi, L. Salmeron-Contreras, I. Herrero-Durá, R. Picó, J. Redondo, V. Sánchez-Morcillo, K. Staliunas, N. Adkins, A. Cebrecos, N. Jiménez et al., Aerosp. Sci. Technol. 73, 300 (2018). 